\title{
Facilitating understanding of mental health problems in GP consultations: a qualitative study using taped-assisted recall
}

\author{
John Cape, Connie Geyer, Chris Barker, Nancy Pistrang, \\ Marta Buszewicz, Christopher Dowrick and Peter Salmon
}

\begin{abstract}
Background

Mental health problems are common in primary care and most are managed solely by the GP. Patients strive to understand their mental health problems, and facilitating patients' understanding may be important in their care, yet little is known about this process in GP consultations.
\end{abstract}

Aim

To explore how patients' understanding of common mental health problems is developed in GP consultations.

\section{Design of study}

Qualitative study.

\section{Setting}

Ten general practices in North Central London.

\section{Method}

Fourteen patients and their GPs were interviewed using the taped-assisted recall (TAR) method, and asked how understanding of the patients' mental health problems had been discussed in a recent consultation. The resulting 42 transcripts of the GP-patient consultations and separate GP and patient TAR interviews were analysed using qualitative thematic and process analytic methods.

\section{Results}

Patients considered understanding their mental health problems to be important, and half reported their GP consultations as helpful in this respect. The process of coming to an understanding was predominantly patient-led. Patients suggested their own explanations, and these were facilitated and focused by the doctors' questioning, listening, validating, and elaborating aspects they considered important. Both doctors and patients experienced constraints on the extent to which developing understanding of problems was possible in GP consultations.

\section{Conclusion}

GPs can help patients understand their mental health problems by recognising patients' own attempts at explanation and helping to shape and develop these.

\section{Keywords}

doctor-patient relations; mental health; primary care; qualitative research.

\section{INTRODUCTION}

Common mental health problems are a significant element of all GPs' workload. ${ }^{1,2}$ The most frequent form of management of such problems is some form of discussion or counselling. ${ }^{3}$ National Institute for Health and Clinical Excellence (NICE) clinical guidelines recommend that psychotropic medications are not used in mild conditions, ${ }^{4}$ and patients with more severe disorders may be reluctant to accept medication, ${ }^{5}$ so that discussion or counselling within the consultation is often the main management option available to GPs. Understanding what aspects of GPs' discussion and counselling of patients might be helpful and how this can be facilitated through GP training is therefore important.

GPs' discussion with, and counselling of, patients with common mental health problems can involve a number of different elements. ${ }^{6-9}$ One of these is helping patients make sense of their problems. Patients with

J Cape, PhD, head of psychology, Camden and Islington NHS Foundation Trust, London. C Geyer, MA, trial manager, Unit for Social and Community Psychiatry, Queen Mary University of London. C Barker, PhD, reader in clinical psychology; $N$ Pistrang, PhD, reader in clinical psychology, Research Department of Clinical, Educational and Health Psychology, University College London. M Buszewicz, MRCGP, MRCPsych, senior lecturer, Research Department of Primary Care and Population Health, University College Medical School, London. C Dowrick, MD, FRCGP, FFPHM, professor of primary mental health care, Division of Primary Care; P Salmon, DPhil, professor of clinical psychology, Division of Clinical Psychology, School of Population, Community and Behavioural Sciences, University of Liverpool, Liverpool.

\section{Address for correspondence}

Professor John Cape, Camden and Islington NHS

Foundation Trust, St Pancras Hospital, 4 St Pancras Way, London, NW1 0PE. E-mail: j.cape@ucl.ac.uk

Submitted: 3 December 2009; Editor's response: 9 February 2010; final acceptance: 11 March 2010.

@British Journal of General Practice

This article was originally online first on 12 October 2010. Cite this article as: Br J Gen Pract 2010; 60: 837-845. Advance online publication. DOI: 10.3399/bjgp10X532567 


\section{How this fits in}

Mental health problems are a significant element of GPs' workload. Little is known about whether and how routine consultations might facilitate patients' understanding of such problems. In this study, some patients found their consultation helpful in understanding their mental health problems. The process of developing understanding in the consultation was predominantly patient led, with patients suggesting their own explanations and GPs facilitating, validating, focusing, and shaping their understanding; GP-led explanations were the exception.

mental health problems actively try to make sense of these problems, ${ }^{10}$ and may use their consultations with GPs to facilitate this, which may in itself have therapeutic value. Assisting those with common mental health problems to find new ways of understanding their symptoms and problems is considered central to psychological change in psychological therapies, and has been associated with clinical benefit over a number of treatments and conditions. $^{9,11,12}$ While the context of GP discussion and counselling is very different to specialist psychological therapy, helping patients make sense of symptoms is consistent with a GP's core role, and may directly promote symptom improvement, as patients' inaccurate conception of their problems may often contribute to their symptoms (for example, a fear of 'going mad' or of 'having a heart attack' in panic disorder)..$^{13}$

The process of facilitating patients' understanding of their problems in GP consultations where patients explicitly present mental health problems has not, as far as we are aware, been previously studied other than with GPs with a specialist interest in such an approach. ${ }^{14,15}$ By contrast, where patients present somatic or medically unexplained symptoms, the process of negotiation between doctor and patient as to the nature of the problem has received considerable research attention. ${ }^{16,17}$

The aim of this qualitative study was to explore how patients' understanding of their mental health problems develops during routine GP consultations, drawing on three sources: audiorecordings of consultations and tape-assisted recall (TAR) interviews with both patients and doctors. In TAR interviews, recordings of the consultation are used to prompt patient and doctor memories of what was said and how they reacted. ${ }^{18,19}$ In a previous study, TAR interviews were used to explore what patients found helpful about mental health consultations. ${ }^{19}$ The present study extends earlier use of the TAR method to include doctor as well as patient TAR interviews and to focus on a specific aspect of the consultation: how patients' understanding of their problems is developed.

\section{METHOD}

\section{Setting and recruitment}

Two hundred and thirty-seven GPs working in North Central London were sent written invitations to participate. Fifteen volunteered, of whom 13 were recruited for the study (see 'Data analysis' section for rationale for ending data collection). The second author approached consecutive adult patients (aged 18-65 years) in the waiting room of these GPs for consent to participate. Consenting patients completed the General Health Questionnaire (GHQ)-12, ${ }^{20}$ and the GP audiorecorded their subsequent consultation.

After each GP surgery, the second author reviewed the audiorecorded consultation and selected consultations where there was significant discussion of mental health problems (defined as containing more than $20 \mathrm{GP}$ or patient statements coded as psychological using an established content analysis scheme), ${ }^{21}$ and where the patient's GHQ-12 was above the threshold score $(\geq 3)$. Consultations where patients' mental health problems were being managed in secondary care were excluded.

\section{Tape-assisted recall interviews}

For each consultation that met study inclusion criteria, the second author approached both GP and patient for interview. Where possible, the GP was interviewed first. Following some open-ended questions about the GP's general impressions of the patient and consultation, the interviewer played back the recorded consultation and, at specific points, asked about:

- the GP's understanding of the patient's mental health problems;

- how the GP thought the patient understood his/her problems;

- what explanations/understanding the GP was trying/hoping to get across to the patient in the consultation; and

- where in the consultation this was taking place.

Following the interview with the GP, the patient was interviewed at home or another convenient place. As with the GP TAR interview, after first asking for general impressions, the interviewer played back the recorded consultation and asked:

- how the patient had thought about their problems before seeing the doctor;

- whether and how the patient's thinking had changed after seeing the doctor; and

- where in the consultation there had been things said that had helped the patient think about their problems differently.

In addition, if the GP had indicated in the GP TAR 
interview places in the recorded consultation where they had tried to explain something to the patient but the patient had not so far commented on this in their TAR interview, the interviewer would ask:

- what the patient thought about and what sense they had made of what the doctor had said at these points in the recorded consultation.

After their TAR interview, patients completed the computerised (PROQSY) version of the Clinical Interview Schedule-Revised (CIS-R), ${ }^{22}$ administered to provide a measure of severity of symptoms. A score of $\geq 12$ indicates a clinically significant level of symptoms. ${ }^{22,23}$

\section{Data analysis}

The data for qualitative analysis were the verbatim transcriptions of the GP-patient consultations and of the GP TAR interviews and patient TAR interviews referring to each consultation.

There were two approaches to the qualitative analysis; these occurred sequentially but were also part of an iterative cycle to refine the analysis. The first approach was to undertake separate thematic analyses of the GPs' and the patients' transcribed TAR interviews to identify central ideas in the GPs' and patients' accounts of developing understanding. The constant comparative method ${ }^{24}$ was used to analyse similarities and differences within and across each set of accounts, and established procedures of thematic qualitative analysis ${ }^{25}$ were used to arrive at a set of common themes.

The second approach was a process analysis, ${ }^{26}$ which focused on each GP-patient pair to analyse how the process of developing understanding unfolded in specific instances from the perspective of both parties. This drew on the transcripts of the consultation and both TAR interviews, enabling links to be made between what was actually said in the consultation, the GP's intentions behind what was said, and the impact on the patient of what was said.

At least two members of the research team analysed all transcripts, and the remaining members acted as analytic auditors of the emerging analysis to ensure reliability. ${ }^{27}$ Data analysis was conducted in parallel with data collection, which ended when little new information was emerging and time demands outweighed the potential benefits of further recruitment.

\section{RESULTS}

\section{Doctor and patient characteristics}

The 13 GP participants were aged $37-57$ years (mean age 41 years); nine were female, four were from minority ethnic backgrounds, and they came from 10 different general practices. One hundred and forty-two of 354 adult patients approached consented to have their consultation recorded; 23 consultations met the study criteria and 16 of these patients agreed to be interviewed. For one consultation, the patient was not subsequently able to complete the TAR interview, and for another consultation the GP was not available, so these two consultations were not analysed. The final study sample was 14 consultations from 11 of the participant GPs (a single consultation from eight GPs, two consultations from each of three GPs). For two GPs no patient was successfully interviewed.

The 14 patients (11 women, 3 men; 13 white, 1 Japanese ; mean age 39 years, range $18-58$ years) had a mean GHQ-12 of 5.91 (standard deviation [SD] 3.20) and mean CIS-R of 17.7 (SD 6.92). All except two had scores of $\geq 12$ on the CIS-R, the threshold indicating a clinically significant level of symptoms. The median length of their consultations was 14 minutes 48 seconds (range 8 minutes 50 seconds to 37 minutes 38 seconds). Only two of the consultations were first consultations for the presenting problem, five were second or subsequent consultations regarding a relatively recently presented problem, and seven were for more longstanding problems, although the GP may not always have seen the patient recently about them (in one case it was 8 months since the patient's last consultation). For each of the 14 patients, there were full transcripts of the consultation, of the GP TAR interview, and of the patient TAR interview; hence a total of 42 transcripts.

\section{Patient views of consultation helpfulness in facilitating understanding}

Seven of the 14 consultations were classified on the basis of patients' comments in their TAR interview as having helped in their understanding of their problems. Comments such as the following were regarded as indicating that the consultation had helped their understanding:

II think when I realised that the way I was feeling is all part of the grieving process and maybe I thought I was ill ... but it's just all things that happen to you.' (P14, patient TAR)

'When she started asking me "how does it affect you?" and then I started to think about so many other things ... since I came here [London] everything has just been working, working, working ... I mean, it really brings you down.' (P170, patient TAR)

The remaining seven patients did not find the consultations added to or changed their understanding of their problems, although they all considered that 
understanding their problems was important and most found the consultations helpful in other ways. No difference was detected between consultations about recently presented problems and more longstanding problems in whether patients considered them helpful in gaining an understanding of their problems.

\section{Process of facilitating understanding}

For most patients who reported their consultations as helpful in understanding their problems, the process was patient led. Only one case was clearly doctor led. In general, doctors did not 'give explanations' leading to patients having new understanding. Rather, they encouraged patients to talk about their problems and come up with their own explanations, either without additional input or through subtle refining and shaping of patients' accounts. There was variation between consultations in the extent to which GPs shaped patients' understanding. Three types of consultation process were distinguished, differing on this continuum of doctors' input, from mostly following patients as they developed their understanding to actively shaping the process:

- encouraging patient reflection;

- focusing and shaping patients' understanding; and

- active guided discovery.

\section{Encouraging patient reflection}

Such consultations were characterised by patients exploring their concerns and doctors listening and making empathic comments indicating their understanding of the patients' accounts. Doctors' encouragement of patient elaboration and reflection was mediated by use of silence, indications of active listening (for example, 'mhm $\mathrm{mhm}$ '), and comments that were supportive ('it's just very difficult') or reflective ('it feels impossible').

For some patients, this process of doctor encouragement of patient reflection did not lead to any new understanding, but helped validate the account with which the patient came into the consultation. It confirmed their pre-existing understanding. This was still reported as helpful:

'Exactly again that my interpretation was confirmed that it was like a bereavement ... a corroboration of my interpretation. Huge bereavement.' (P01, patient TAR)

For other patients, the GP's facilitation of their talk about their problems led to a new perspective. Talking things through helped patients come up with a different perspective as a result of what one patient referred to as a process of 'explaining things to myself'. This was often not a completely new understanding of their problems but a 'refreshed' understanding (as in the patient needed to 'remind' him/herself of this):

'It's just having a person listening to you, and I think the more you talk the more you find that sense a bit yourself. And telling someone else, you know, that makes sense to me then.' (P14, patient TAR)

'I know what I was thinking at the time, which is that it seemed like something of a revelation at the same time as ... I don't mean revelation in the sense of somebody telling me something that you don't already know but in the sense of somebody telling me something that you go, "Yes!" That's so the way it is.' (P243, patient TAR)

GPs were aware of this process and occasionally commented explicitly on it:

I think if you push them in the right direction patients will often make most of the running. Um and it's just a question of keeping them going as they sort it out for themselves really.' (P14, GP TAR)

However, encouraging patients to talk and reflect on their problems was not always associated with increased understanding: there was also evidence of these processes in consultations that patients considered as not adding to or changing their understanding of their problems. Hence, while encouragement of patient exploration and selfreflection may have been a sufficient facilitator of new understanding for some patients, for others, additional factors were necessary for this to take place.

\section{Focusing and shaping patients' understanding}

In addition to encouraging patients to talk, doctors also usually subtly focused, shaped, refined and extended patients' understanding in the consultations, which patients reported as helping understanding. They did this through the questions they chose to ask, and what they chose to reflect back to the patient, to summarise, and to elaborate.

\section{Asking questions}

Asking questions early in the consultation regarding patients' concerns or thoughts about physical symptoms that they presented was used to prompt patients to extend their accounts from the purely symptomatic to emotional concerns, anxieties, and wider psychosocial issues:

GP: 'And what is it that's worrying you when that [heart pounding] happens?'

Patient: 'I think basically it's because my mind is just going into overdrive, thinking about different 
things, you know, people that l've lost and things.' (P14, consultation)

'That's a technique I use of trying to get people to identify what's on their minds when they're experiencing a physical symptom that I think is stress related. So I'm trying to get at the source of the stress and of course, and then she brings it up pretty quickly doesn't she, you know "People l've lost and things", I mean she immediately responds doesn't she? So she is, she is thinking about the losses is, isn't she?' (P14, GP TAR)

However, questions of this kind were not always successful in prompting patients to extend their accounts and bring in other concerns that would give a broader context to their presenting symptoms. Patients would sometimes just continue to discuss their physical symptoms or add further physical symptoms:

GP: 'When you're lying there trying to get back off to sleep, do you have any particular thoughts, anxiety feelings, how are you feeling when you're trying to get back to sleep?'

Patient: 'No, what I seem to have is a sort of uh it's almost like a physical feeling rather than you know thoughts or emotions or whatever.' (P201, consultation)

'I thought it was a reasonable question ... But l've got a straight answer to it which is that I very rarely have these sort of emotional things going around.' (P201, patient TAR)

Questions were also used to help patients explore potentially relevant factors in their mental health problems or distress. Sometimes the GPs' questions would draw on prior knowledge of the patient and what might be relevant to the patient's distress:

GP: 'When did you last feel okay?'

Patient: 'Oh, it's been a long time.'

GP: 'What, before your Mum died?'

Patient: 'I mean my Mum was sick for about 6 or

7 years and ...'

GP: 'Did you cry about that? ...' [patient becomes tearful] It's ok. It's normal.' (P114, consultation)

At other times, where the patient was less well known to the GP or this was a new presentation of mental health problems, the questions might cover a wide area of possible concerns, one or more of which would prompt the patient to a new perspective. In the next example, the GP's questions prompted the patient to think of several relevant factors, both in their current life and in their past, which they continued to think about following the consultation. Only some of this new thinking had been expressed in the consultation itself, and the GP was consequently not aware of this or the beneficial impact of the consultation on the patient:

'But when she started asking me like do you have friends and how does it make you sad and how does it make you feel you're like, "oh actually! Oh actually!" and it just starts to pop up in your head.' (P170, patient TAR)

\section{Reflecting back and summarising}

Reflecting back and summarising was used to pick out and emphasise elements of the patient's account that the doctor felt it was useful to highlight. At times, the doctor's summary added no more than an emphasis on some aspect or aspects of what the patient had said, serving as a cue to the patient that the doctor thought this important, which might lead to possible further exploration by the patient of this aspect:

'So there's quite a few changes, there's some changes up ahead that I can see in terms of your work and obviously you need to sort out this problem with your immigration and having broken up your relationship recently.' (P137, GP, consultation)

Commonly, summarising would be accompanied by a perspective which the doctor considered might be important or helpful in extending the patient's understanding. The doctor's added perspective was grafted on to the account that the patient had been giving, so that it followed the patient's lead:

Patient: 'Yes but of course you see I was under this great illusion that we would have an ongoing friendship.'

GP: '... I think it will take time. It's less than a year already. I think it is like a bereavement and there will be stages.' (P01, consultation)

The doctor explained her reason for giving the patient this added perspective as follows:

'... she feels he has completely destroyed her life ... I see my function as trying to get her to let go a bit.' (P01, GP TAR)

On occasions, GPs' summarising would make use of the patient's words, but in such a way as to give the patient a new perspective (Box 1). 


\section{Box 1. Example of focusing and shaping.}

\section{Consultation}

P243: '... it's [muscular dystrophy] just quite painful and tiring and depressing.' GP10: 'yeah, yeah.'

P243: '... and l've been really cold since I came back, just can't seem to get warm so it's just very diff-, very depressing. Sorry [laughs].'

GP10: 'It's not easy to put up with, this, is it? (no) You're obviously somebody, you like to keep very active and getting around the place and doing what you want to do.'

P243: 'I just don't want it to be on top of me (yes) and it feels like it's on top of me.'

GP10: 'We've got to reverse that, haven't we? We can't get rid of the (no) dystrophy, but you can be on top of it rather than the other way (yeah), rather than the other way round somehow.' (P243, consultation)

\section{GP TAR interview}

GP10: 'I assume that she was telling me that it feels that it's on top of her, that the dystrophy is ruining her life, is taking over her life rather than her having control of her life and coping with the dystrophy ... But by saying "it's on top of me" she's implying there could be an opportunity to be on top of it, so it's about wanting to move her to think about how she can be on top of it rather than not on top of it.' (P243, GP TAR)

\section{Box 2. Example of guided discovery.}

\section{Consultation}

P198: 'I panic less.'

GP: 'What do you do to panic less?'

P198: 'Just keep calm and try to breathe.'

GP8: 'Okay. Talk me through it.'

P198: 'I just go [inhales] like that and I try not to shake ... I just start swallowing a lot (aha) and then just get up and I (ok).'

GP8: 'And then what do you do after that, do you know?'

P198: 'I open my mouth and I go, like you told me to.'

GP8: 'And does it work?'

P198: 'Yeah.' (P198, consultation)

\section{GP TAR interview}

GP8: I'm trying to demonstrate the fact that what her symptoms are showing is she can cope with it and she is doing something about it ... so she knows she's feeling a bit panicky and the choking is not going to kill her ... She panics less because she's got a better understanding of what's going on, so therefore she can manage it better in a stressful situation ... "all I need to do is deep-breathe".' (P198, GP TAR)

\section{Patient TAR interview}

Interviewer (I):

'So what do you think he was trying to tell you in that little segment that we just listened to?'

P198: 'That there's nothing wrong with me.'

I: 'And what do you think he is trying to achieve with that?'

P198: 'Not just saying it but believe it as well.'

I: 'And did that happen for you?'

P198: 'Well it has, it's getting there. It's not fully there but I'd say about $80 \%$, it's getting there.' (P198, patient TAR)

\section{Active guided discovery}

One consultation, considered by the patient as contributing to increased understanding of their problems, was different from the others in that the process of the patient coming to a different understanding was almost entirely doctor led. The doctor described taking an active role in educating the patient about their hyperventilation and panic symptoms. This included teaching breathing methods and then using a process of guiding the patient in thinking through what their success in controlling hyperventilation meant for an understanding of their symptoms (Box 2).

While there were examples in other consultations of GP comments that were more active in guiding patients towards particular understandings or explanations, these comments were in the context of an overall patient-led approach. They added to and extended patients' understanding as described above. Therefore, if patients' responses to GPs' comments indicated that they did not fit or make sense to the patient, they were not pursued by the doctor.

\section{Constraints in developing understanding}

In the TAR interviews, both GPs and patients mentioned constraints and limitations on the extent to which developing understanding about problems was possible in the study consultations. These were raised both for consultations that patients felt had been helpful in furthering their understanding, and for consultations that had not contributed to understanding. Constraints were classified into those related to contextual, patient, doctor, and doctor-patient relationship factors.

Contextual constraints. Both doctors and patients commented on constraints inherent in brief consultations. Doctors' comments were generally about the limitations of what can be achieved in a single consultation, with a perspective that future consultations would give opportunities to expand relevant issues:

'I just felt there is an issue I think of how much you can tackle in any consultation, or indeed to some extent a set of consultations.' (P301, GP TAR)

'I just think she mentioned so many different things, I didn't know which thing to focus on ... And I will see her again so I don't have to do everything in one consultation. She'll come back at some point ...' (P243, GP TAR)

Some patients were more acutely concerned about time, feeling there wouldn't be enough time to talk about and take in what the doctor was saying: 
I'm so pressured ... and that I've got a hell of a lot that I have got to go over and talk about, I'm talking and sometimes not listening absolutely specifically, specifically to her because there's so much I need to talk about.' (P01, patient TAR)

Both patients and doctors commented on a perceived lack of capacity of GPs to deal with mental health problems and this was a key issue in two consultations. In both these consultations, the patient commented on having come with limited expectations as to what could be achieved, and the doctor appeared to agree with this assessment. In one of these, the patient had extensive prior treatment from counsellors and psychiatrists, while the other was on a waiting list for cognitive behaviour therapy:

'Well I was trying to bump it off onto the psychologist ... I think it's too deep rooted for someone like me to deal with.' (P286, GP TAR)

GPs at times explicitly restricted their exploration of understanding of patients' problems on the grounds that this would be more appropriately conducted by another doctor (the patient's usual doctor) or counsellor or therapist involved with the patient's care.

Patient constraints. Some patients talked about anxiety and embarrassment as a constraint on their exploring their understanding of their problems with the doctor. This was often accompanied by feeling unsure whether it was appropriate to talk about personal problems with a GP, or guilt for taking up too much of their time. This constraint was only mentioned by patients who reported no impact on their understanding of their problems from the consultation:

'Maybe I'm not really saying what I need to say because I'm nervous and embarrassed about the situation so I just concentrate on the symptoms and the medication.' (P201, patient TAR)

GP remarks about patient constraints varied according to the patient. Particular patients were perceived as too fragile, or too fixed on their own explanation of the problem for it to be appropriate to raise other possible ideas. Postponing bringing up alternative ideas about problems until a future consultation, because of patient capacity or vulnerability, was a strategy GPs also mentioned:

'But I feel I must almost be more cautious than I would be with somebody else because of her very fragile mental state.' (P01, GP TAR)

GP: 'I think psychological factors are far more powerful than most of us, and certainly most of our patients, give credit for.'

Interviewer: 'Did you feel there was any scope to renegotiate that with her in the consultation?'

GP: '... Not at that time. I think what was really important was to validate her concern that there could be a physical reason.' (P143, GP TAR)

Doctor constraints. Constraints related to doctor factors were less likely to receive comment from either GPs or patients. GPs, while listening to the consultations in their TAR interviews, would at times comment on how they pursued a line of irrelevant medical questioning or too quickly suggested a referral to a counsellor which distracted the patient from pursuing an understanding of their problems. They speculated that this was the 'easier' option, rather than sticking with patients' exploration of their distress. In one case, the GP suggested that this arose from his sense of frustration that he felt unable to help:

'So there I am trying to medicalise her ... "maybe we'll just give you some thyroxine and make you better" ... So it's sort of palming her off when I know it's not really her thyroid ... I feel a bit hands tied behind my back because I don't know what else to do. I don't really have the time or the skills.' (P286, GP TAR)

Doctor-patient relationship constraints. One patient commented on the familiar light-hearted style of her relationship with the doctor as a possible constraint on further exploration of understanding of her problems. This style was apparent from the consultation transcript and appeared to cover a sense of frustration from both doctor and patient about lack of progress in understanding or in therapeutic strategies:

'I don't know just I think partly Dr X and I, it's kind of - the sort of relationship we've built up we're kind of - we have a bit of banter together and kind of - like we sort of laugh a lot at things ... But it's not a good thing you know and I, it's almost like it's this kind of little charade but it's this thing we go through every time when we have these consultations.' (P286, patient TAR)

\section{DISCUSSION}

\section{Summary of main findings}

Patients considered that understanding their mental health problems was important, and half of the 
sample of patients reported their consultations as helpful in developing this understanding. The process of developing understanding was predominantly patient led, with patients suggesting their own explanations and GPs actively responding to their lead. Within this, there was a range of input from GPs, ranging from corroboration of patients' expressed explanations, through elicitation and corroboration of preformed but unexpressed ideas, to more formative work in developing, facilitating, and shaping patients' understanding of their problems. However, all this work by the GP facilitated the development of explanations given by the patient, rather than fashioning explanations for the patient.

Notwithstanding this evidence of GPs facilitating patients' understanding of their mental health problems, both doctors and patients also experienced constraints on the extent to which this was possible in GP consultations. Commonly voiced constraints included patient factors (for example, anxiety and embarrassment) and consultation context (for example, time and capacity), with doctor and doctor-patient relationship factors less commonly mentioned.

\section{Strengths and limitations of the study}

The study design drew on three sources of data for each consultation: the consultation transcript, the GP TAR interview, and the patient TAR interview. What was said by patient and doctor in the consultation could be triangulated and compared with the intention of the speaker and effects on the listener. When the object of study (patients' understanding) is subjective, but the vehicle influencing that understanding (the consultation) is objective, methods that examine both are needed.

Selection procedures for the study excluded consultations about common mental health problems where patients did not talk psychologically about their problems. In addition, study GPs were self-selected volunteers who may have been more experienced and positive about working with patients with mental health problems and specifically more interested in ways of understanding patients' problems. ${ }^{28}$ Thus, there are questions about the generalisability of the study findings to the full range of GPs and patients with mental health problems.

As the study was cross-sectional and focused on single consultations, it was not possible to elucidate how patients' understanding might develop over a series of consultations. It is possible that understandings that some patients brought with them to the study consultations were the result of previous work by the same, or another GP.

\section{Comparison with existing literature}

Although patients with mental health problems are known to actively try to make sense of these problems, ${ }^{10}$ and facilitating more helpful understanding of their problems is central to the study of psychological therapies, ${ }^{11}$ there is little prior research on the process of developing patients' understanding of explicitly presented mental health problems in GP consultations.

A historic tradition of enquiry, associated with Michael Balint, has been centrally concerned with how patients' understanding of their problems might be developed by GPS. ${ }^{14,29}$ A central finding from this literature is the importance of the doctor understanding how patients view and experience their world, in order to develop their understanding. The need for a similar collaborative process has been recognised in relation to GP consultations in a related area: medically unexplained symptoms.$^{30}$ The patientled process of developing understanding in the present study, with GPs following but then also subtly shaping patients' understanding, is consistent with this earlier work. Empathic understanding by the GP of the patient's problems is considered important by patients with mental health problems, ${ }^{6,21}$ and may be a critical and even necessary precondition of GPs helping patients to develop an understanding of their problems.

Eliciting patients' views and engaging with patients' understanding of their symptoms to help them understand their condition, rather than just providing standard explanations, has been a theme in the general medical communication literature,$^{31}$ and there is evidence that this process may improve health outcomes. ${ }^{32}$ Thus, the consultation strategies for developing understanding of mental health problems may not be essentially different from those used to develop understanding for other problems in general practice.

The constraints, mentioned by both GPs and patients, about the extent to which GP consultations can be a place to develop patients' understanding of their mental health problems, are all too familiar from research on other aspects of GP consultations. Consultation time and GPs' limited capacity are 'hardy perennials', ${ }^{33,34}$ while patient reticence due to embarrassment and anxiety is particularly common in mental health consultations. ${ }^{35}$

\section{Implications for future research and clinical practice}

The present study was a preliminary investigation into the process of facilitating patient understanding of mental health problems in routine GP consultations. This needs to be explored in a wider range of consultations, to see whether other approaches to 
developing understanding might occur. Exploration of how patients' understanding develops longitudinally over the course of a series of GP consultations is also needed.

From this preliminary descriptive research, it is premature to draw recommendations for clinical practice. However, this study suggests that developing patients' understanding of their explicitly presented mental health problems does occur in the routine GP consultations of interested GPs using the standard consultation skills of careful listening, connecting with patients' understanding of their symptoms, and shaping and extending this understanding, and that patients can experience this as helpful.

\section{Funding body}

North Central London Research Consortium (NoCLoR) (06MHS83)

\section{Ethical approval}

Ethical approval was obtained from Camden and Islington Community Local Research Ethics Committee (06/Q0511/60)

\section{Competing interests}

The authors have stated that there are none.

\section{Acknowledgements}

We would like to thank Sara Hutchings and the GPs and patients who participated.

\section{Discuss this article}

Contribute and read comments about this article on the Discussion Forum: http://www.rcgp.org.uk/bjgp-discuss

\section{REFERENCES}

1. Goldberg DP Lecrubier Y. Form and frequency of mental disorders across centres. In: Ustun B, Sartorius N (eds). Mental illness in general health care: an international study. Chichester: WHO \& John Wiley, 1995: 323-334.

2. Toft T, Fink P, Oernboel E, et al. Mental disorders in primary care: prevalence and co-morbidity among disorders. Results from the Functional Illness in Primary Care (FIP) study. Psychol Med 2005; 35(8): 1175-1184

3. MaGPie Research Group. The treatment of common mental health problems in general practice. Fam Pract 2006; 23(1):53-59.

4. National Institute for Clinical Excellence. Depression: management of depression in primary and secondary care. London: NICE, 2004.

5. Paykel ES, Hart D, Priest RG. Changes in public attitudes to depression during the Defeat Depression Campaign. Br J Psychiatry 1998; 173: 519-522.

6. Cape J, Barker C, Buszewicz M, Pistrang N. General practitioner psychological management of common emotional problems (I): definitions and literature review. Br J Gen Pract 2000; 50(453): 313-318.

7. Roter DL, Stewart M, Putnam SM, et al. Communication patterns of primary care physicians. JAMA 1997; 277(4): 350-356.

8. Linden M, Christof T, Rentzsch C. Contents of general practitioner-patient consultations in the treatment of depression. Gen Intern Med 2008; 23(10): 1567-1570.

9. Cape J, Barker C, Buszewicz M, Pistrang N. General practitioner psychological management of common emotional problems (II): research agenda for evidence-based practice. Br J Gen Pract 2000; 50(454): 396-400.

10. Williams B, Healey D. Perceptions of illness causation among new referrals to a community mental health team: 'explanatory model' or 'exploratory map'? Soc Sci Med 2001; 53(4): 465-476.

11. Power M, Brewin C. Transformation of meaning in psychological therapies. New York, NY: Wiley, 1997.

12. Hubble MA, Duncan BL, Miller SD (eds). The heart and soul of change: what works in therapy? Washington, DC: American Psychological Association, 1999.
13. Clark DM, Salkovskis PM, Ost L-G, et al. Misinterpretation of body sensations in panic disorder. J Consult Clin Psychol 1997; 65(2): 203-213.

14. Balint E, Norell JS. Six minutes for the patient: interactions in general practice consultations. London: Tavistock, 1973.

15. Launer J, Lindsey C. Training for systemic general practice: a new approach from the Tavistock Clinic. Br J Gen Pract 1997; 47(420): 453-456.

16. Salmon P. Patients who present physical symptoms in the absence of physical pathology: a challenge to existing models of doctor-patient interaction. Patient Educ Couns 2000; 39(1): 105-113.

17. Ring A, Dowrick C, Humphris G, Salmon P. Do patients with unexplained physical symptoms pressure GPs for somatic treatment? A qualitative study. BMJ 2004; 328(7447): 1057-1060.

18. Elliott R, Shapiro DA. Brief structured recall: a more efficient method for studying significant therapy events. Br J Med Psychol 1988; 61(Pt 2): 141-153.

19. Buszewicz M, Pistrang N, Barker C, et al. Patients' experiences of GP consultations for psychological problems: a qualitative study. $\mathrm{Br} J \mathrm{Gen}$ Pract 2006; 56(528): 496-503.

20. Goldberg D, Williams P. A user's guide to the General Health Questionnaire. Windsor: NFER-Nelson, 1998

21. Cape J. Psychological treatment of emotional problems by general practitioners. Br J Med Psychol 1996; 69(Pt 2): 85-99.

22. Lewis G. Assessing psychiatric disorder with a human interviewer or a computer. J Epidemiol Community Health 1994; 48(2): 207-210.

23. McManus S, Meltzer H, Brugha T, et al. (eds). Adult psychiatric morbidity in England, 2007: results of a household survey. London: The NHS Information Centre for Health and Social Care, 2009.

24. Strauss A, Corbin J. Basics of qualitative research: techniques and procedures for developing grounded theory. 2nd edn. Newbury Park, CA: Sage, 1998.

25. Barker C, Pistrang N, Elliott R. Research methods in clinical psychology: an introduction for students and practitioners. 2nd edn. Chichester: Wiley, 2002.

26. Pistrang N, Barker C, Rutter C. Social support as conversation: Analysing breast cancer patients' interactions with their partners. Soc Sci Med 1997; 45(5): 773-782.

27. Stiles WB. Evaluating qualitative research. Evid Based Ment Healt 1999; 2(4): 99-101.

28. Cape J, Morris E, Burd M, Buszewicz M. Complexity of GPs explanations about mental health problems: development, reliability and validity of a measure. Br J Gen Pract 2008; 58(551): 403-410.

29. Balint M. The doctor, his patient and the illness. 2nd edn. London: Pitman Medical, 1964.

30. Salmon P. Conflict, collusion or collaboration in consultations about medically unexplained symptoms: the need for a curriculum of medical explanation. Patient Educ Couns 2007; 67(3): 246-254.

31. Stewart M, Brown J, Weston W, et al. Patient-centered medicine: transforming the clinical method. Thousand Oaks, CA: Sage, 1995.

32. Street RL, Makoul G, Arora NK, Epstein RM. How does communication heal? Pathways linking clinician-patient communication to health outcomes. Patient Educ Couns 2009; 74(3): 295-301.

33. Dowrick C, Gask L, Hughes J, et al. General practitioners' views on reattribution for patients with medically unexplained symptoms: a questionnaire and qualitative study. BMC Fam Pract 2008; 9: 46.

34. Peters S, Rogers A, Salmon P, et al. What do patients choose to tell their doctors? Qualitative analysis of potential barriers to reattributing medically unexplained symptoms. J Gen Intern Med 2009; 24(4): 443-449.

35. Cape J, McCulloch Y. Patients' reasons for not presenting emotional problems in general practice consultations. Br J Gen Pract 1999; 49(448): 875-879. 